Interfaces and Free Boundaries 15 (2012), 167-180

DOI 10.4171/IFB/299

\title{
A free-boundary problem for concrete carbonation: Front nucleation and rigorous justification of the $\sqrt{t}$-law of propagation
}

\author{
TOYOHIKO AIKI \\ Department of Mathematics, Faculty of Science, Japan Women's University \\ E-mail: aikit@fc.jwu.ac.jp
}

ADRIAN MUNTEAN

CASA - Centre for Analysis, Scientific computing and Applications, Department of Mathematics and Computer Science, Institute for Complex Molecular Systems (ICMS), Eindhoven University of Technology, The Netherlands

E-mail: a.muntean@tue.nl

[Received 8 June 2011 and in revised form 1 October 2012]

\begin{abstract}
We study a one-dimensional free-boundary problem describing the penetration of carbonation fronts (free reaction-triggered interfaces) in concrete. Using suitable integral estimates for the free boundary and involved concentrations, we reach a twofold aim:

(1) We fill a fundamental gap by justifying rigorously the experimentally guessed $\sqrt{t}$ asymptotic behavior. Previously we obtained the upper bound $s(t) \leqslant C^{\prime} \sqrt{t}$ for some constant $C^{\prime}$; now we show the optimality of the rate by proving the right nontrivial lower estimate, i.e., there exists $C^{\prime \prime}>0$ such that $s(t) \geqslant C^{\prime \prime} \sqrt{t}$.

(2) We obtain weak solutions to the free-boundary problem for the case when the measure of the initial domain vanishes. In this way, we allow for the nucleation of the moving carbonation front - a scenario that until now was open from the mathematical analysis point of view.
\end{abstract}

2010 Mathematics Subject Classification: Primary 35Q35.

Keywords: Large-time behavior; free-boundary problem; concrete carbonation; integral estimates.

\section{Introduction}

\subsection{Background}

Environmental impact on concrete parts of buildings results in a variety of unwanted chemical and chemically-induced mechanical changes. The bulk of these changes leads to damaging and destabilization of the concrete itself or of the reinforcement embedded in the concrete. One important destabilization factor is the drop in $\mathrm{pH}$ near the steel bars induced by carbonation of the alkaline constituents; see for instance [15, 16, 26] and [23, 24] for technical details. Note however that, depending on the precise situation, carbonation is not necessarily a bad think to happen to a building material, on top of this it can also play an important role in $\mathrm{CO}_{2}$ sequestration as mentioned in [12] and references cited therein. The destabilization is caused by atmospheric carbon dioxide diffusing in the dry parts and reacting in the wet parts of the concrete pores. The phenomenon is considered as one of the major processes inducing corrosion in concrete. A particular feature of carbonation is the formation of macroscopic sharp reaction interfaces or thin reaction layers that progress into the unsaturated concrete-based materials. The deeper cause for the formation of these patterns is not quite clear, although the major chemical and physical reasons seem to be known. 
Mathematically, the proposed model is a coupled system of semi-linear partial differential equations posed in a single 1D moving domains. The moving interface (front position in 1D) is assumed to be triggered by a fast chemical reaction - the carbonation reaction. Non-linear transmission conditions of Rankine-Hugoniot type are imposed across the inner boundary that separates the carbonated regions from the uncarbonated ones. The movement of the carbonated region is determined via a non-local dynamics law.

Note that our research is in spirit very much aligned with the mathematical investigations by $\mathrm{R}$. Natalini and co-workers on the restoration of historical monuments; see for instance [5, 13]. Related mathematical approaches of similar reaction-diffusion scenarios have been reported, for instance, in $[8,13,14]$, focussing on the fast-reaction asymptotics. Note however that the precise structure of our model has not yet been derived by such arguments, in spite of repeated attempts [9, 17].

\subsection{Aim of the paper}

As key objectives we wish to prove that a carbonation front can nucleate based on our model equations and that its penetration depth asymptotics for large times can be predicted. Of course, other questions like understanding general motions of macroscopic sharp reaction fronts in concrete [especially in the presence of corners] would be highly interesting both mathematically and from the civil engineering point of view, but this is for the moment out of reach due to that fact that we do not know yet the right boundary conditions in $2 \mathrm{D}$ and $3 \mathrm{D}$. Possible routes towards this might be provided by applying a phase-field methodology.

Note that a couple of decades ago, it was observed experimentally that the carbonation penetration depth versus time curve ( say $s(t)$ vs. $t$ ) behaves like $s(t)=C \sqrt{t}$ for sufficiently large times $t>0$ (with $C$ a positive constant). Consequently, many fitting arguments solely based on this experimental law were used to predict the large-time behavior of carbonation fronts in real structures, a theoretical justification of the $\sqrt{t}$-law being lacking until now.

A fundamental result stating when the $\sqrt{t}$-law is expected to hold is really needed especially if one looks to the large amount of current engineering literature reporting on $t^{\beta}$ behaviors, where $\beta$ is typically a parameter fluctuating around $\frac{1}{2}$. The summary of experimentally expected asymptotic behaviors [for various types of cements and various boundary (environmental) conditions] reported in Table 2.2, pp. 30-31 [23] shows luck of agreement and general confusion.

This is the place where our paper contributes: We want to fill this gap by justifying rigorously the experimentally discovered asymptotic behavior; see, e.g., [24] for the case when one can ensure that carbonation does not significantly affect the porosity of the concrete.

\subsection{Basic carbonation scenario - a moving one-phase approach}

We study a one-dimensional free boundary problem system arising in the modeling of concrete carbonation problem. We consider that the concrete occupies the infinite interval $(0, \infty)$ and that there exists a sharp interface $x=s(t), t>0$ separating the carbonated from the uncarbonated zone. The whole process can be seen as a solid-solid phase change; see the two colors in Fig. 1 (left). One color points out to $\mathrm{CaCO}_{3}$ (carbonated phase), while the other one indicates $\mathrm{Ca}(\mathrm{OH})_{2}$ (uncarbonated phase). The zone of interest is only one of the solid phases, namely the carbonated zone. We denote it by $Q_{s}(T)$ and, in mathematical terms, this is defined by $Q_{s}(T):=\{(t, x)$ : $0<t<T, 0<x<s(t)\}$ for some $T>0$. Throughout this paper $u$ and $v$ denote the mass 
concentrations of $\mathrm{CO}_{2}$ in air and water, respectively. In this framework, $u$ and $v$ satisfy the following system $\mathrm{P}=\mathrm{P}\left(s_{0}, u_{0}, v_{0}, g, h\right)(1.1) \sim(1.7)$ :

$$
\begin{aligned}
& u_{t}-\left(\kappa_{1} u_{x}\right)_{x}=f(u, v) \quad \text { in } Q_{s}(T), \\
& v_{t}-\left(\kappa_{2} v_{x}\right)_{x}=-f(u, v) \quad \text { in } Q_{s}(T), \\
& u(t, 0)=g(t), v(t, 0)=h(t) \quad \text { for } 0 \leqslant t \leqslant T, \\
& s^{\prime}(t)=\psi(u(t, s(t))) \quad \text { for } 0<t<T, \\
& -\kappa_{1} u_{x}(t, s(t))=\psi\left(u(t, s(t))+s^{\prime}(t) u(t, s(t)) \quad \text { for } 0<t<T,\right. \\
& -\kappa_{2} v_{x}(t, s(t))=s^{\prime}(t) v(t, s(t)) \quad \text { for } 0<t<T, \\
& s(0)=s_{0} \text { and } u(0, x)=u_{0}, v(0, x)=v_{0} \quad \text { for } 0<x<s_{0},
\end{aligned}
$$

where $\kappa_{1}$ (resp. $\kappa_{2}$ ) is a diffusion constant of $\mathrm{CO}_{2}$ in air (resp. water), $f(u, v):=\beta(\gamma v-u)$ is an effective Henry's law, where $\beta$ and $\gamma$ are positive constants, $g$ and $h$ are given functions corresponding to boundary conditions for $u$ and $v$, respectively, $\psi(r):=\alpha\left|[r]^{+}\right|^{p}$ for $r \in R$ describes the rate of the carbonation reaction, where $p \geqslant 1$ and $\alpha$ is a positive constant ${ }^{\ddagger} . s_{0} \geqslant 0$ is the initial position of the free boundary, while $u_{0}$ and $v_{0}$ are the initial concentrations.

Looking to Fig. 1, one could wonder whether a one-dimensional setting is proper or not when dealing with this problem. To defend the 1D approach, we just consider a scenario away from corners and have in mind that the actual measurement of the thickness of the width of the colorless zone is essentially $1 \mathrm{D}$. This measurement of the carbonated width is called phenolphtalein test.

First mathematical models with free boundaries for describing the concrete carbonation process have been proposed by Muntean and Böhm in [18], where the first mathematical results concerning the global existence and uniqueness of weak solutions as well as the stability of the solutions with respect to data and parameters have been investigated. Recently, we have improved their results by focussing a reduced free-boundary model still able to capturing the basic features of the carbonation process; see [1,2] for the list of the new theorems on the existence and uniqueness of weak solutions to P. This model is in some sense minimal: It includes the transport of species (diffusion), their averaged transfer across air-water interfaces (the Henry law), as well as fast reaction (with an indefinitely large chemical compound - "the concrete"). We have used further the advantageous structure of the reduced model to study the large-time behavior of the penetration depths. Basically, we started to wonder whether the experimentally known $\sqrt{t}$-law

$$
s(t)=\bar{C} \sqrt{t} \text { for } t>0,
$$

where $\bar{C}$ is a positive constant, is true or not [20]. Let us comment a bit on the context: It was shown in [26, 193-199] that the carbonation front behaves like a similarity solution to a one-phase Stefanlike problem [21]. Using matched-asymptotics techniques, the fast-reaction limit (for large Thiele moduli) done in [17] for a reaction-diffusion system also led to a $\sqrt{t}$-behavior of the carbonation front supporting experimental results from, e.g., $[15,16]$. On the other hand, experimental results from [23] indicate that, depending on the type of the cement, a variety of $t^{\beta}$ front behaviors with $\beta \neq$ $\frac{1}{2}$ are possible; see also Fig. 1 (right) where we point out that our model approaches qualitatively the expected experimental behavior. Furthermore, Souplet, Fila and collaborators (compare [10,

\footnotetext{
$\$$ The exponent $p$ is sometimes called order of the chemical reaction, while the parameter $\alpha$ is just a proportionality constant. Its sensitivity with respect to the model output $(s, u, v)$ has been studied numerically in [19].

We do not know yet how the speed of the carbonation front is affected by the local geometry (e.g., corners).
} 

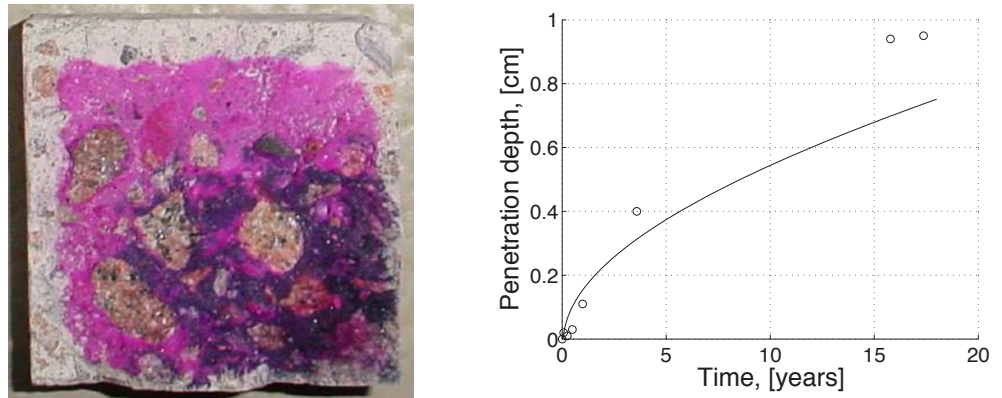

FIG. 1. (Left) Typical result of the phenolphthalein test on a partially carbonated sample (Courtesy of Prof. Dr. Max Setzer, University of Duisburg-Essen, Germany). The dark region indicates the uncarbonated part, while the brighter one points out the carbonated part. The two regions are separated by a sharp interface moving inwards the material. In this colorimetric test, this macroscopic interface corresponds to a drop in $\mathrm{pH}$ below 10. (Right) Computed interface positions vs. measured penetration depths [19]. This indicates how the mathematical model approximates the real situation.

25]) have shown that, under certain conditions, non-homogeneous Stefan-like problem can lead to asymptotics like $s(t) \sim t^{\frac{1}{3}}$. Somehow, the major question remains:

What is the correct asymptotics of the carbonation front propagation?

The main result of our preliminary investigations (based on the reduced FBP) is reported in [3] and supports the fact that

$$
s(t) \rightarrow \infty \text { as } t \rightarrow \infty \text { and } s(t) \leqslant C^{\prime} \sqrt{t} \text { for } t \geqslant 0,
$$

where $C^{\prime}$ is a positive constant. Moreover, in this paper we establish as main result (see Theorem 2.3) a lower bound on the position of the free boundary $s$ as follows: For some positive constant $c$

$$
s(t) \geqslant c \sqrt{t} \text { for } t \geqslant 0 .
$$

This estimate combined with the corresponding lower one would immediately guarantee the correctness of the $\sqrt{t}$-law from a mathematical modeling point of view. In Section 2 we derive the missing lower bound. Our results here are not trivial extensions of the analysis presented in [3] - conceptually better ideas were needed to improve in an essential manner the available results. In particular, note that since, generally, $k_{1} \gg k_{2}$ and $\gamma v \neq u$, the system (1.1)-(1.7) cannot be reduced to a scalar equation, where the use of Green functions representation $[6,21]$ would very much facilitate the obtaining of non-trivial lower bounds on concentrations, and hence, on the free boundary velocity. Furthermore, by using the similar method as the one used in the proof of (1.8), we can construct a weak solution to P satisfying $s_{0}=0$. It is worth mentioning that Fasano and Primicerio (cf., e.g., [11]) have investigated a one-phase Stefan problem when the measure of the initial domain vanishes. In their proof the comparison principle is used in an essential manner. However, for our problem $\mathrm{P}$ we do not have any comparison theorem for the free boundary. Our idea here is to develop a method to obtain improved uniform estimates for solutions and then use these estimates to prove the existence of weak solutions for the case $s_{0}=0$ (nucleation of the carbonation front). This program is realized in section 3. There are neither physical nor mathematical reasons to believe that uniqueness of weak solutions for the case $s_{0}=0$ would not hold. However, since our fixing-domain technique is not applicable anymore, the uniqueness seems to be difficult to prove. 


\section{Large-time behavior of the free boundary}

In order to give a statement of our result on the large-time behavior of (weak) solutions, we consider the problem P posed in the cylindrical domain $Q(T):=(0, T) \times(0,1)$. To this end, we use the following change of variables:

Let

$$
\bar{u}(t, y)=u(t, s(t) y) \quad \text { and } \quad \bar{v}(t, y)=v(t, s(t) y) \quad \text { for }(t, y) \in Q(T) .
$$

Then, it holds that

$$
\begin{aligned}
& \bar{u}_{t}-\frac{\kappa_{1}}{s^{2}} \bar{u}_{y y}-\frac{s^{\prime}}{s} y \bar{u}_{y}=f(\bar{u}, \bar{v}) \quad \text { in } Q(T), \\
& \bar{v}_{t}-\frac{\kappa_{2}}{s^{2}} \bar{v}_{y y}-\frac{s^{\prime}}{s} y \bar{v}_{y}=-f(\bar{u}, \bar{v}) \quad \text { in } Q(T), \\
& \bar{u}(0, t)=g(t), \bar{v}(0, t)=h(t) \quad \text { for } 0<t<T, \\
& s^{\prime}(t)=\psi(\bar{u}(t, 1)) \quad \text { for } 0<t<T, \\
& -\frac{\kappa_{1}}{s(t)} \bar{u}_{y}(t, 1)=s^{\prime}(t) \bar{u}(t, 1)+s^{\prime}(t) \quad \text { for } 0<t<T, \\
& -\frac{\kappa_{2}}{s(t)} \bar{v}_{y}(t, 1)=s^{\prime}(t) \bar{v}(t, 1) \quad \text { for } 0<t<T, \\
& s(0)=s_{0}, \bar{u}(0, y)=\bar{u}_{0}(y), \bar{v}(0, y)=\bar{v}_{0}(y) \quad \text { for } 0<y<1,
\end{aligned}
$$

where $\bar{u}_{0}(y)=u_{0}\left(s_{0} y\right)$ and $\bar{v}_{0}(y)=v_{0}\left(s_{0} y\right)$ for $y \in[0,1]$.

For simplicity, we introduce some notations as follows: $H:=L^{2}(0,1), X:=\left\{z \in H^{1}(0,1)\right.$ : $z(0)=0\}, X^{*}$ is the dual space of $X$,

$$
V(T):=L^{\infty}(0, T ; H) \cap L^{2}\left(0, T ; H^{1}(0,1)\right)
$$

and

$$
V_{0}(T):=V(T) \cap L^{2}(0, T ; X),
$$

and $(\cdot, \cdot)_{H}$ and $\langle\cdot, \cdot\rangle_{X}$ denote the usual inner product of $H$ and the duality pairing between $X$ and $X^{*}$, respectively.

First of all, we define a weak solution of $\mathrm{P}\left(s_{0}, u_{0}, v_{0}, g, h\right)$. To do this, we use a similar concept of weak solution as the one introduced in [1].

DEFinition 2.1 Let $s$ be a function on $[0, T]$ and $u$, $v$ be functions on $Q_{s}(T)$ for $0<T<\infty$, and $\bar{u}$ and $\bar{v}$ be functions defined by (2.1). We call that a triplet $\{s, u, v\}$ is a weak solution of $\mathrm{P}$ on $[0, T]$ if the conditions $(\mathrm{S} 1) \sim(\mathrm{S} 5)$ hold:

(S1) $s \in W^{1, \infty}(0, T)$ with $s>0$ on $[0, T],(\bar{u}, \bar{v}) \in\left(W^{1,2}\left(0, T ; X^{*}\right) \cap V(T) \cap L^{\infty}(Q(T))\right)^{2}$.

(S2) $\bar{u}-g, \bar{v}-h \in L^{2}(0, T ; X), u(0)=u_{0}$ and $v(0)=v_{0}$.

(S3) $s^{\prime}(t)=\psi\left(u(t, s(t))\right.$ for a.e. $t \in[0, T]$ and $s(0)=s_{0}$.

$$
\begin{aligned}
& \int_{0}^{T}\left\langle\bar{u}_{t}, z\right\rangle_{X} d t+\int_{Q(T)} \frac{\kappa_{1}}{s^{2}} \bar{u}_{y} z_{y} d y d t+\int_{0}^{T} \frac{s^{\prime}}{s}(\bar{u}(\cdot, 1)+1) z(\cdot, 1) d t \\
&=\int_{Q(T)}\left(f(\bar{u}, \bar{v})+\frac{s^{\prime}}{s} y \bar{u}_{y}\right) z d y d t \quad \text { for } z \in V_{0}(T) .
\end{aligned}
$$




$$
\begin{aligned}
& \int_{0}^{T}\left\langle\bar{v}_{t}, z\right\rangle_{X} d t+\int_{Q(T)} \frac{\kappa_{2}}{s^{2}} \bar{v}_{y} z_{y} d y d t+\int_{0}^{T} \frac{s^{\prime}}{s} \bar{v}(\cdot, 1) z(\cdot, 1) d t \\
&=\int_{Q(T)}\left(-f(\bar{u}, \bar{v})+\frac{s^{\prime}}{s} y \bar{v}_{y}\right) z d y d t \quad \text { for } z \in V_{0}(T) .
\end{aligned}
$$

Moreover, let $s$ be a function on $[0, \infty)$, and $u$ and $v$ be functions on $Q_{s}:=\{(t, x) \mid t>0,0<x<$ $s(t)\}$. We say that $\{s, u, v\}$ is a weak solution of $\mathrm{P}$ on $[0, \infty)$ if for any $T>0$ the triplet $\{s, u, v\}$ is a weak solution of $\mathrm{P}$ on $[0, T]$.

Before recalling our results concerning the global existence and uniqueness of weak solutions to $\mathrm{P}$ on the time interval $[0, T], T>0$, we give the following assumptions for the involved data and model parameters:

(A1) $f(u, v)=\beta(\gamma v-u)$ for any $(u, v) \in \mathbf{R}^{2}$ where $\beta$ and $\gamma$ are positive constants.

(A2) $g, h \in W_{l o c}^{1,2}([0, \infty)) \cap L^{\infty}(0, \infty)$, and $g \geqslant 0$ and $h \geqslant 0$ on $(0, \infty)$.

(A3) $u_{0} \in L^{\infty}\left(0, s_{0}\right)$ and $v_{0} \in L^{\infty}\left(0, s_{0}\right)$ with $u_{0} \geqslant 0$ and $v_{0} \geqslant 0$ on $\left(0, s_{0}\right)$.

THEOREM 2.2 (cf. [1, Theorems 1.1 and 1.2, Lemma 4.1]) If (A1) (A3) hold, then P has one and only one weak nonnegative solution on $[0, \infty)$.

The next theorem is the main result of this paper.

Theorem 2.3 If $g(t)=g_{*}, h(t)=h_{*}$ for $t \in[0, \infty)$, where $g_{*}$ and $h_{*}$ are positive constants with $\gamma h_{*}=g_{*}$, and (A1) and (A3) hold, then there exists a positive constant $c$ such that

$$
s(t) \geqslant c \sqrt{t} \quad \text { for } t \geqslant 0 .
$$

The proof of Theorem 2.3 relies on the following technical lemma containing integral estimates for both the concentrations and position of the free boundary, positive and uniformly bounded concentrations, and also, that an energy-like inequality holds.

LEMma 2.4 (cf. [3, Lemmas 3.2, 3.3 and 3.4]) If (A1) (A3) hold, then a weak solution $\{s, u, v\}$ on $[0, \infty)$ satisfies

$$
\begin{aligned}
\int_{0}^{s(t)} x u(t) d x+\frac{1}{2}|s(t)|^{2} & +\kappa_{1} \int_{0}^{t} u(\tau, s(\tau)) d \tau+\int_{0}^{s(t)} x v(t) d x+\kappa_{2} \int_{0}^{t} \int_{0}^{s(\tau)} v_{x}(\tau, x) d x d \tau \\
= & \int_{0}^{s_{0}} x u_{0} d x+\frac{1}{2}\left|s_{0}\right|^{2}+\int_{0}^{s_{0}} x v_{0} d x+\kappa_{1} \int_{0}^{t} g(\tau) d \tau \quad \text { for } t \geqslant 0
\end{aligned}
$$


and

$$
\begin{aligned}
& \frac{1}{2} \int_{0}^{s(t)}\left|u(t)-g_{*}\right|^{2} d x+\frac{\gamma}{2} \int_{0}^{s(t)}\left|v(t)-h_{*}\right|^{2} d x+\frac{1}{2} \int_{0}^{t}\left|s^{\prime}(\tau)\right|^{1+2 / p} d \tau \\
& +\kappa_{1} \int_{0}^{t} \int_{0}^{s(\tau)}\left|u_{x}(\tau)\right|^{2} d x d \tau+\gamma \kappa_{2} \int_{0}^{t} \int_{0}^{s(\tau)}\left|v_{x}(\tau)\right|^{2} d x d \tau \\
& \leqslant \frac{1}{2} \int_{0}^{s_{0}}\left|u_{0}-g_{*}\right|^{2} d x+\frac{\gamma}{2} \int_{0}^{s_{0}}\left|v_{0}-h_{*}\right|^{2} d x \\
& \quad+\int_{0}^{t} s^{\prime}(\tau)\left(\frac{1}{2}\left|g_{*}\right|^{2}+g_{*}+\frac{\gamma}{2}\left|h_{*}\right|^{2}\right) d \tau \quad \text { for } t \geqslant 0 .
\end{aligned}
$$

Moreover, if positive numbers $g^{*}$ and $h^{*}$ satisfying $u_{0} \leqslant g^{*}, v_{0} \leqslant h^{*}$ on $\left[0, s_{0}\right], g \leqslant g^{*}, h \leqslant h^{*}$ on $[0, \infty)$ and $g^{*}=\gamma h^{*}$, then it holds that

$$
0 \leqslant u \leqslant g^{*}, 0 \leqslant v \leqslant h^{*} \text { on } Q_{s}
$$

\subsection{Proof of Theorem 2.3.}

In this section, we give the proof of our main result.

Proof. Let $\{s, u, v\}$ be a weak solution of $\mathrm{P}$ on $[0, \infty)$, and $g^{*}$ and $h^{*}$ be positive constants defined in Lemma 2.4. First, Lemma 2.4 implies that

$$
\begin{aligned}
& \gamma \kappa_{2} \int_{0}^{t} \int_{0}^{s(\tau)}\left|v_{x}(\tau)\right|^{2} d x d \tau \\
& \quad \leqslant \frac{1}{2} \int_{0}^{s_{0}}\left(\left|u_{0}-g_{*}\right|^{2}+\gamma\left|v_{0}-h_{*}\right|^{2}\right) d x+\int_{0}^{t} s^{\prime}(\tau)\left(\frac{1}{2}\left|g_{*}\right|^{2}+g_{*}+\frac{\gamma}{2}\left|h_{*}\right|^{2}\right) d \tau \\
& \quad \leqslant \frac{1}{2} \int_{0}^{s_{0}}\left(\left|u_{0}-g_{*}\right|^{2}+\gamma\left|v_{0}-h_{*}\right|^{2}\right) d x+\left(\frac{1}{2}\left|g_{*}\right|^{2}+g_{*}+\frac{\gamma}{2}\left|h_{*}\right|^{2}\right)\left(s(t)-s_{0}\right) \quad \text { for } t \geqslant 0 .
\end{aligned}
$$

Hence, there is a positive constant depending on $u_{0}, v_{0}, g_{*}, h_{*}$ and $s_{0}$ such that

$$
\int_{0}^{t} \int_{0}^{s(\tau)}\left|v_{x}(\tau)\right|^{2} d x d \tau \leqslant C_{1}+C_{1} s(t) \quad \text { for } t \geqslant 0
$$

Next, on account of Lemma 2.4 we see that

$$
\begin{aligned}
\int_{0}^{s(t)} x u(t) d x & +\frac{1}{2}|s(t)|^{2}+\kappa_{1} \int_{0}^{t} u(\tau, s(\tau)) d \tau+\int_{0}^{s(t)} x v(t) d x+\kappa_{2} \int_{0}^{t} \int_{0}^{s(\tau)} v_{x}(\tau, x) d x d \tau \\
& =\int_{0}^{s_{0}} x u_{0} d x+\frac{1}{2}\left|s_{0}\right|^{2}+\int_{0}^{s_{0}} x v_{0} d x+\kappa_{1} \int_{0}^{t} g(\tau) d \tau \\
& \geqslant \kappa_{1} g_{*} t \quad \text { for } t \geqslant 0 .
\end{aligned}
$$

Here, we note that

$$
u(t, s(t))=\left(\frac{s^{\prime}(t)}{\alpha}\right)^{1 / p} \quad \text { for } t \geqslant 0
$$


Then, by putting $M=\max \left\{g^{*}, h^{*}\right\}$ we obtain

$$
\begin{aligned}
\kappa_{1} g_{*} t \leqslant 2 M \int_{0}^{s(t)} x d x+\frac{1}{2}|s(t)|^{2} & +\frac{\kappa_{1}}{\alpha^{1 / p}} \int_{0}^{t}\left(s^{\prime}(\tau)\right)^{1 / p} d \tau \\
& +\kappa_{2}\left(\int_{Q_{s}(t)}\left|v_{x}\right|^{2} d x d \tau\right)^{1 / 2}\left(\int_{Q_{s}(t)} d x d \tau\right)^{1 / 2} \quad \text { for } t \geqslant 0 .
\end{aligned}
$$

In order to apply the Young inequality,

$$
a b \leqslant C_{p, \varepsilon} a^{p}+\varepsilon b^{1-1 / p} \quad \text { for } a, b \geqslant 0, p \geqslant 1 \text { and } \varepsilon>0,
$$

where $C_{p, \varepsilon}$ is a positive constant depending on $\varepsilon$, let $\varepsilon>0$. Then, it is clear that

$$
\begin{aligned}
\frac{\kappa_{1}}{\alpha^{1 / p}} \int_{0}^{t}\left(s^{\prime}(\tau)\right)^{1 / p} d \tau & \leqslant \frac{\kappa_{1}}{\alpha^{1 / p}}\left(\int_{0}^{t} s^{\prime}(\tau) d \tau\right)^{1 / p} t^{1-1 / p} \\
& \leqslant \frac{\kappa_{1}}{\alpha^{1 / p}} s(t)^{1 / p} t^{1-1 / p} \\
& \leqslant \varepsilon t+C_{p, \varepsilon} \frac{\kappa_{1}^{p}}{\alpha} s(t) \quad \text { for } t \geqslant 0 .
\end{aligned}
$$

By taking $\varepsilon=\frac{1}{4} \kappa_{1} g_{*}$, we have

$$
\frac{\kappa_{1}}{\alpha^{1 / p}} \int_{0}^{t}\left(s^{\prime}(\tau)\right)^{1 / p} d \tau \leqslant \frac{1}{4} \kappa_{1} g_{*} t+C_{2} s(t),
$$

where $C_{2}=C_{p, \varepsilon}\left(\frac{\kappa_{1}}{\alpha^{1 / p}}\right)^{p}$, and

$$
\begin{aligned}
\kappa_{2}\left(\int_{Q_{s}(t)}\left|v_{x}\right|^{2} d x d \tau\right)^{1 / 2}\left(\int_{Q_{s}(t)} d x d \tau\right)^{1 / 2} & \leqslant \kappa_{2} C_{1}^{1 / 2}(1+s(t))^{1 / 2} t^{1 / 2} s(t)^{1 / 2} \\
& \leqslant \frac{1}{4} \kappa_{1} g_{*} y+C_{3}\left(s(t)+s(t)^{2}\right) \quad \text { for } t \geqslant 0
\end{aligned}
$$

where $C_{3}$ is some positive constant.

From the above inequalities we can get

$$
\frac{1}{2} \kappa_{1} g_{*} t \leqslant\left(M+\frac{1}{2}+C_{3}\right)|s(t)|^{2}+\left(C_{2}+C_{3}\right) s(t) \quad \text { for } t \geqslant 0 .
$$

Now, let $t \geqslant 1$. In this case by applying the Young inequality, again, we see that

$$
\left(C_{2}+C_{3}\right) s(t) \leqslant \frac{1}{4} \kappa_{1} g_{*} t+C_{4}|s(t)|^{2} \quad \text { for } t \geqslant 1,
$$

where $C_{4}$ is some positive constant so that we have

$$
\frac{1}{2} \kappa_{1} g_{*} t \leqslant\left(M+\frac{1}{2}+C_{3}\right)|s(t)|^{2}+C_{4}|s(t)|^{2}+\frac{1}{4} \kappa_{1} g_{*} t \quad \text { for } t \geqslant 0 .
$$


Thus it is easy to get

$$
\begin{gathered}
\frac{1}{4} \kappa_{1} g_{*} t \leqslant\left(M+1+C_{3}+C_{4}\right)|s(t)|^{2}, \\
\left(\frac{\kappa_{1} g_{*}}{4\left(M+1+C_{3}+C_{4}\right)} t\right)^{1 / 2} \leqslant s(t) \quad \text { for } t \geqslant 1 .
\end{gathered}
$$

In case $0 \leqslant t \leqslant 1$, we have $s_{0} \sqrt{t} \leqslant s(t)$.

Therefore, by putting $v_{0}=\min \left\{s_{0},\left(\frac{\kappa_{1} g_{*}}{4\left(M+1+C_{3}+C_{4}\right)}\right)^{1 / 2}\right\}$ we conclude that

$$
v_{0} \sqrt{t} \leqslant s(t) \quad \text { for } t \geqslant 0 \text {. }
$$

\section{Nucleation of the carbonation front: The case $s_{0}=0$}

The aim of this section is to prove a result concerning the existence of weak solutions to $\mathrm{P}$ for the case $s_{0}=0$. This is the case when the free boundary starts off moving precisely from the outer boundary [exposed to $\mathrm{CO}_{2}$ ]. This refers to the stage often called in Landau's theory of phase transitions as the nucleation phase.

Before giving the statement of the theorem, we denote for simplicity

$$
C_{0}((0, T] ; X)=\left\{z \in C([0, T]: X): z=0 \text { on }\left[0, \delta_{z}\right) \text { for some } \delta_{z}>0\right\} .
$$

THEOREM 3.1 Let $T>0$, and $g$ and $h$ be functions on $[0, T]$ satisfying $g, h \in W^{1,2}(0, T)$ and $g(t) \geqslant g_{0}>0$ and $h \geqslant 0$ for $t \in[0, T]$, where $g_{0}$ is a given positive constant. Then under (A1) there exists a triplet $\{s, u, v\}$ of functions such that $s \in W^{1, \infty}(0, T), s(0)=0, s(t)>0$ for $t \in(0, T]$, $\bar{u}, \bar{v} \in L^{\infty}(Q(T)), \bar{u}-g, \bar{v}-h \in L^{2}(0, T ; X), \bar{u}, \bar{v} \in C((0, T] ; H), \bar{u}, \bar{v} \in W_{l o c}^{1,2}\left((0, T] ; X^{*}\right)$,

$$
\begin{gathered}
s^{\prime}(t)=\psi(\bar{u}(t, 1)) \quad \text { for a.e. } t \in[0, T], \\
\int_{0}^{T}\left\langle\bar{u}_{t}, z\right\rangle_{X} d t+\int_{Q(T)} \frac{\kappa_{1}}{s^{2}} \bar{u}_{y} z_{y} d y d t+\int_{0}^{T} \frac{s^{\prime}}{s} z(\cdot, 1) d t \\
=\int_{Q(T)}\left(f(\bar{u}, \bar{v})-\frac{s^{\prime}}{s} y \bar{u}_{y}\right) z d y d t \quad \text { for } z \in C_{0}((0, T] ; X), \\
\int_{0}^{T}\left\langle\bar{v}_{t}, z\right\rangle_{X} d t+\int_{Q(T)} \frac{\kappa_{2}}{s^{2}} \bar{v}_{y} z_{y} d y d t \\
=-\int_{Q(T)}\left(f(\bar{u}, \bar{v})+\frac{s^{\prime}}{s} y \bar{v}_{y}\right) z d y d t \quad \text { for } z \in C_{0}((0, T] ; X),
\end{gathered}
$$

where $\bar{u}$ and $\bar{v}$ are functions defined by (2.1).

Proof. First, let $\left\{s_{0 n}\right\}$ be a sequence satisfying $s_{0 n}>0$ for each $n$ and $s_{0 n} \rightarrow 0$ as $n \rightarrow \infty$ and put $u_{0 n}=g(0)$ and $v_{0 n}=h(0)$ on $\left[0, s_{0 n}\right]$. Then, Theorem 2.2 guarantees that $\mathrm{P}\left(s_{0 n}, u_{0 n}, v_{0 n}, g, h\right)$ has a unique weak solution $\left\{s_{n}, u_{n}, v_{n}\right\}$ on $[0, T]$. Here, we denote by $\bar{u}_{n}$ and $\bar{v}_{n}$ the functions defined by (2.1) with $s=s_{n}, u=u_{n}$ and $v=v_{n}$ for each $n$. Since we can take positive constants 
$g^{*}$ and $h^{*}$ such that $g \leqslant g^{*}$ and $h \leqslant h^{*}$ on $[0, T], u_{0 n} \leqslant g^{*}$ and $v_{0 n} \leqslant h^{*}$ on $\left[0, s_{0 n}\right]$ for $n$ and $g^{*}=\gamma h^{*}$, Lemma 2.4 implies that

$$
0 \leqslant u_{n} \leqslant g^{*}, 0 \leqslant v_{n} \leqslant h^{*} \quad \text { on } Q_{s_{n}}(T) \text { for any } n .
$$

By (S3) and this shows that $\left|s_{n}^{\prime}(t)\right| \leqslant \psi\left(g^{*}\right)$ for $t \in[0, T]$ and $n$ so that the set $\left\{s_{n}\right\}$ is bounded in $W^{1, \infty}(0, T)$. Clearly, there exists a positive constant $L_{1}$ such that $0 \leqslant s_{n}(t) \leqslant L_{1}$ for $t \in[0, T]$ and $n$.

Next, the following estimate is a direct consequence of [1, Lemma 4.2]: For each $n$

$$
\begin{aligned}
& \kappa_{1} \int_{0}^{t} \int_{0}^{s_{n}(\tau)}\left|u_{n x}\right|^{2} d x d \tau+\kappa_{2} \int_{0}^{t} \int_{0}^{s_{n}(\tau)}\left|v_{n x}\right|^{2} d x d \tau \\
& \leqslant 2\left(C_{f}^{2}+1\right) \int_{0}^{t} \int_{0}^{s_{n}(\tau)}\left(\left|u_{n}(\tau)-g(\tau)\right|^{2}+\left|v_{n}(\tau)-h(\tau)\right|^{2}\right) d x d \tau \\
&+2 \int_{0}^{t} s_{n}(\tau)\left(|f(g(\tau), h(\tau))|^{2}+\left|g_{\tau}(\tau)\right|^{2}+\left|h_{\tau}(\tau)\right|^{2}\right) d \tau \\
& \quad+\int_{0}^{t} s_{n}^{\prime}(\tau)\left(3|g(\tau)|^{2}+|g(\tau)|+|h(\tau)|^{2}\right) d \tau \quad \text { for } t \in[0, T],
\end{aligned}
$$

where $C_{f}:=\beta \gamma$. Because of the boundedness of $\left\{s_{n}\right\}$ and (3.4) there exists a positive constant $M_{2}$ such that

$$
\int_{0}^{T} \int_{0}^{s_{n}(\tau)}\left|u_{n x}\right|^{2} d x d \tau+\int_{0}^{T} \int_{0}^{s_{n}(\tau)}\left|v_{n x}\right|^{2} d x d \tau \leqslant M_{2} \text { for } n .
$$

Then, easily, we can obtain that $\left\{\bar{u}_{n y}\right\}$ and $\left\{\bar{v}_{n y}\right\}$ are bounded in $L^{2}(Q(T))$.

From now on we provide the estimate from below for the free boundary as follows. To do so from Lemma 2.4 it follows that

$$
\begin{aligned}
\kappa_{1} g_{0} t \leqslant & \int_{0}^{s_{n}(t)} x u_{n}(t) d x+\frac{1}{2}\left|s_{n}(t)\right|^{2}+\kappa_{1} \int_{0}^{t} u_{n}\left(\tau, s_{n}(\tau)\right) d \tau \\
& +\int_{0}^{s_{n}(t)} x v_{n}(t) d x+\kappa_{2} \int_{0}^{t} \int_{0}^{s_{n}(\tau)} v_{n x}(\tau, x) d x d \tau \\
= & : J_{1 n}(t)+J_{2 n}(t)+J_{3 n}(t)+J_{4 n}(t)+J_{5 n}(t) \quad \text { for } t \geqslant 0 \text { and } n .
\end{aligned}
$$

Here, it is obvious that

$$
\left.\begin{array}{l}
J_{1 n}(t)+J_{4 n}(t) \leqslant \frac{1}{2}\left(g^{*}+h^{*}\right)\left|s_{n}(t)\right|^{2} \\
J_{5 n}(t) \leqslant \kappa_{2} M_{2}^{1 / 2}\left(t s_{n}(t)\right)^{1 / 2}
\end{array}\right\} \text { for } t \in[0, T] \text { and } n .
$$

Similarly to (2.2), by using (1.4) we observe that

$$
J_{3 n}(t) \leqslant \frac{\kappa_{1}}{\alpha^{1 / p}} \int_{0}^{t}\left|s_{n}^{\prime}(\tau)\right|^{1 / p} d \tau \leqslant \frac{\kappa_{1} T^{1-1 / p}}{\alpha^{1 / p}} s_{n}(t)^{1 / p} \text { for } t \in[0, T] \text { and } n .
$$


From the above inequalities we have

$$
\begin{aligned}
\kappa_{1} g_{0} t & \leqslant \frac{g^{*}+h^{*}+1}{2} s_{n}(t)^{2}+\frac{\kappa_{1} T^{1-1 / p}}{\alpha^{1 / p}} s_{n}(t)^{1 / p}+\kappa_{2}\left(M_{2} T\right)^{1 / 2} s_{n}(t)^{1 / 2} \\
& \leqslant\left(\frac{g^{*}+h^{*}+1}{2} L_{1}^{2-\mu}+\frac{\kappa_{1} T^{1-1 / p}}{\alpha^{1 / p}} L_{1}^{1 / p-\mu}+\kappa_{2}\left(M_{2} T\right)^{1 / 2} L_{1}^{1 / 2-\mu}\right) s_{n}(t)^{\mu} \\
& =: M_{3} s_{n}(t)^{\mu} \text { for } t \in[0, T] \text { and } n,
\end{aligned}
$$

where

$$
\mu:=\min \{1 / p, 1 / 2\}
$$

so that

$$
s_{n}(t) \geqslant v_{1} t^{1 / \mu} \text { for } t \in[0, T] \text { and } n,
$$

where $v_{1}$ is a positive constant independent of $n$.

As next step, we wish to estimate the time derivative of $\bar{u}_{n}$. Let $\delta>0$ and $\eta \in L^{2}(\delta, T ; X)$. Then (S4) implies that

$$
\begin{aligned}
& \left|\int_{\delta}^{T}\left\langle\bar{u}_{n t}(t), \eta(t)\right\rangle_{X} d t\right| \\
& \leqslant\left|\int_{\delta}^{T} \frac{\kappa_{1}}{s_{n}^{2}(t)}\left(\bar{u}_{y}(t), \eta_{y}(t)\right)_{H} d t\right|+\left|\int_{\delta}^{T}\left(\frac{s_{n}^{\prime}(t)}{s_{n}(t)} \bar{u}_{n}(t, 1)+\frac{s_{n}^{\prime}(t)}{s_{n}(t)}\right) \eta(t, 1) d t\right| \\
& \quad+\left|\int_{\delta}^{T}\left(f\left(\bar{u}_{n}(t), \bar{v}_{n}(t)\right), \eta(t)\right)_{H} d t\right|+\left|\int_{\delta}^{T} \frac{s_{n}^{\prime}(t)}{s_{n}(t)}\left(y \bar{u}_{n y}(t), \eta(t)\right)_{H} d t\right| \\
& =: I_{1 n}+I_{2 n}+I_{3 n}+I_{4 n} .
\end{aligned}
$$

Obviously, on account of (3.5) it holds that

$$
\begin{gathered}
I_{1 n} \leqslant \kappa_{1} \int_{\delta}^{T} \frac{1}{v_{1}^{2} t^{2 / \mu}}\left|\bar{u}_{n y}(t)\right|_{H}\left|\eta_{y}(t)\right|_{H} d t \\
\leqslant \frac{\kappa_{1}}{v_{1}^{2} \delta^{2 / \mu}}\left|\bar{u}_{n y}\right|_{L^{2}(\delta, T ; H)}\left|\eta_{y}(t)\right|_{L^{2}(\delta, T ; H)} ; \\
I_{2 n} \leqslant \frac{\psi\left(g^{*}\right)}{\mu_{1} \delta^{1 / \mu}}\left(\left|\bar{u}_{n}\right|_{L^{2}\left(0, T ; H^{1}(0,1)\right)}+T^{1 / 2}\right)|\eta|_{L^{2}(\delta, T ; X)} ; \\
I_{3 n} \leqslant \beta\left(\gamma h^{*}+g^{*}\right) T^{1 / 2}|\eta|_{L^{2}(\delta, T ; X)} ; \\
I_{4 n} \leqslant \frac{\psi\left(g^{*}\right)}{v_{1} \delta^{1 / \mu}}\left|\bar{u}_{n y}\right|_{L^{2}(0, T ; H)}|\eta|_{L^{2}(\delta, T ; X)} \quad \text { for } n .
\end{gathered}
$$

Hence, the set $\left\{\bar{u}_{n t}\right\}$ and $\left\{\bar{v}_{n t}\right\}$ are bounded in $L^{2}\left(\delta, T ; X^{*}\right)$ for each $\delta>0$.

From these estimates we can take a subsequence $\left\{n_{j}\right\} \subset\{n\}$ satisfying $s_{n_{j}} \rightarrow s$ weakly* in $W^{1, \infty}(0, T)$ and $C([0, T])$, and $\bar{u}_{n_{j}} \rightarrow \bar{u}$ and $\bar{v}_{n_{j}} \rightarrow \bar{v}$ weakly* in $L^{\infty}(Q(T))$ and weakly in 
$L^{2}\left(0, T ; H^{1}(0,1)\right)$, in $C([\delta, T] ; H)$ and weakly in $W^{1,2}\left(\delta, T ; X^{*}\right)$ for each $\delta>0$ as $j \rightarrow \infty$, where $s \in W^{1, \infty}(0, T), \bar{u}, \bar{v} \in L^{\infty}(Q(T)), \bar{u}-g, \bar{v}-h \in L^{2}(0, T ; X)$ and

$$
\bar{u}, \bar{v} \in C((0, T] ; H) \cap W_{l o c}^{1,2}\left((0, T] ; X^{*}\right) .
$$

By (3.5) we have $s(t)>0$ for $t>0$. Also, $s(0)=0$.

In order to complete the proof of the Theorem, it is necessary to show that (3.2), (3.3) and (3.1) hold. Let $\eta \in C_{0}((0, T] ; X)$. Then $\eta=0$ on $[0, \delta]$ for some $\delta>0$. By taking $z=\eta$ in (S4) we infer that

$$
\begin{aligned}
\int_{\delta}^{T}\left\langle\bar{u}_{n_{j} t}(t), \eta(t)\right\rangle_{X} d t+\int_{\delta}^{T} & \frac{\kappa_{1}}{s_{n_{j}}^{2}(t)}\left(\bar{u}_{n_{j} y}(t), \eta_{y}(t)\right)_{H} d t \\
& +\int_{\delta}^{T}\left(\frac{s_{n_{j}}^{\prime}(t)}{s_{n_{j}}(t)} \bar{u}_{n_{j}}(t, 1)+\frac{s_{n_{j}}^{\prime}(t)}{s_{n_{j}}(t)}\right) \eta(t, 1) d t \\
& =\int_{\delta}^{T}\left(f\left(\bar{u}_{n_{j}}(t), \bar{v}_{n_{j}}(t)\right)+\frac{s_{n_{j}}^{\prime}(t)}{s_{n_{j}}(t)} y \bar{u}_{n_{j} y}(t), \eta(t)\right)_{H} d t \quad \text { for } j .
\end{aligned}
$$

Elementary calculations yield:

$$
\bar{u}_{n_{j}}(\cdot, 1) \rightarrow \bar{u}(\cdot, 1) \text { in } L^{4}(\delta, T) \text { as } j \rightarrow \infty
$$

so that

$$
\int_{\delta}^{T} \frac{s_{n_{j}}^{\prime}(t)}{s_{n_{j}}(t)} \bar{u}_{n_{j}}(t, 1) \eta(t, 1) d t \rightarrow \int_{\delta}^{T} \frac{s^{\prime}(t)}{s(t)} \bar{u}(t, 1) \eta(t, 1) d t \quad \text { as } j \rightarrow \infty .
$$

Moreover, we can obtain $s_{n_{j}}^{\prime} \rightarrow s^{\prime}$ in $L^{4}(\delta, T)$ as $j \rightarrow \infty$ and

$$
\int_{\delta}^{T}\left(\frac{s_{n_{j}}^{\prime}(t)}{s_{n_{j}}(t)} y \bar{u}_{n_{j} y}(t), \eta(t)\right)_{H} d t \rightarrow \int_{\delta}^{T}\left(\frac{s^{\prime}(t)}{s(t)} y \bar{u}_{y}(t), \eta(t)\right)_{H} d t \text { as } j \rightarrow \infty .
$$

Therefore, we can prove that (3.2) holds. Similarly, (3.3) is valid. Finally, by (3.6) we get (3.1). Thus the proof of this theorem has been finished.

\section{More practical comments}

Before using in the engineering practice the $\sqrt{t}$ information for forecasting purposes, the practitioner should be aware of the fact that its validity is closely related to the validity of the underlying free-boundary model $\mathrm{P}$.

- Relying on our working experience with such FBPs for carbonation (based on, e.g., [19]), we can say that $\mathrm{P}$ captures well accelerated carbonation tests, but it may not be suitable for predicting the evolution of carbonation scenarios under natural exposure conditions.

- If the aggressive chemical process (the carbonation reaction in this case) turns our to affect the microstructure (i.e., the pores of the material), then significant deviations from $\sqrt{t}$-like penetration depths become suddenly possible. Mathematically, changes in porosity intimately lead to the occurrence of memory effects (cf., e.g., [4]) that make any large-time asymptotics very difficult to understand and a rigorous forecast seems to be out of reach. 
- In civil engineering terms, one case state that Portland cements are most likely prone to satisfy the $\sqrt{t}$-law, while cements containing pozzolan, fly ash, etc. will deviate from this [22, 24]. For Portland cements, the expected behavior of the front is

$$
\left(s(t)-c^{*} \sqrt{t}\right)=o(t)
$$

for large values of $t$, but without a quantitative control yet on the size and structure of the material constant $c^{*}>0$.

\section{More mathematical comments}

The assumptions of Theorem 2.3 may seem restrictive due to the use of constant Dirichlet values for the two driving concentrations $\left(\mathrm{CO}_{2}\right.$ and $\left.\mathrm{Ca}(\mathrm{OH})_{2}\right)$. Note however that slight changes in these Dirichlet values are expected to produce slight changes in the position of the carbonation front, therefore also in its $t \rightarrow \infty$ asymptotics. Essentially, our model is stable with respect to the choice of data and of parameters. On the other hand, special time-asymptotics of Dirichlet data (or of flux boundary conditions) as well as the presence of a time-evolving porosity [7] of concrete lead to mathematical challenges, e.g., when dealing with memory terms. We will treat some of these issues in a forthcoming publication.

\section{Acknowledgments}

We thank the reviewers for their useful comments, they significantly improved the quality of our manuscript. The initial phase of this research has been supported by the German Science Foundation (DFG), special program SP 1122.

\section{REFERENCES}

1. Aiki, T. And Muntean, A., Existence and uniqueness of solutions to a mathematical model predicting service life of concrete structures, Adv. Math. Sci. Appl. 19 (2009), 109-129. Zbl1181. 35344 MR2553472

2. AiKi, T. AND MunteAn, A., On uniqueness of a weak solution of one-dimensional concrete carbonation problem, Discr. Cont. Dyn. Syst. Ser. A 29 (2010), 1345-1365. Zbl1210. 35296 MR2773187

3. Aiki, T. And Muntean, A., Large time behavior of solutions to concrete carbonation problem, Comm. Pure Appl. Anal. 9 (2010), 1117-1129. Zbl1202.35362 MR2645987

4. Antonic, N., Memory effects in homogenisation: Linear second-order equations, Archive for Rational Mechanics and Analysis 125 (1993), 1-24. Zb10791. 35009 MR1241285

5. Aregba-Driollet, D. , Diele, F. And Natalini, R., A mathematical model for the $\mathrm{SO}_{2}$ aggression to calcium carbonate stones: Numerical approximation and asymptotic analysis, SIAM J. Appl. Math. 64 (2004), 1636-1667. Zbl1126.65072 MR2084204

6. Cannon, J. R., The One-Dimensional Heat Equation, vol. 23 of Encyclopedia of Mathematics and Its Applications, Addison-Wesley, Menlo Park, California, 1984. Zb10567. 35001 MR0747979

7. Chadam, J. And Ortoleva, P., A mathematical problem in geochemistry: The reaction-infiltration instability, Rocky Mountain J. Math. 21 (1991), 631-643. Zb10850.76220 MR1121531

8. DU, Y. AND LIN, Z., Spreading-vanishing dichotomy in the diffusive logistic model with a free boundary, SIAM J. Math. Anal. 42 (2010), 377-405. Zbl1219. 35373 MR2607347

9. Evans, J. D., Fernandez, A. And Muntean, A., Single and two-scale sharp-interface models for concrete arbonation - Asymptotics and numerical approximation, Multiscale Model. Simul. 10 (2012), 874-905. Zbl1255.35138 MR3022025 
10. Fila, M. AND Souplet, P., Existence of global solutions with slow decay and unbounded free boundary for a superlinear Stefan problem, Interfaces and Free Boundaries 3 (2001), 337-344. Zbl1006. 35103 MR1843590

11. Fasano, A. And Primicerio, M., General free-boundary problems for the heat equation. II, J. Math. Anal. Appl. 58 (1977), 202-231. Zbl0355. 35037 MR0487017

12. Galan, I, Andrade, C., Mora, P. and Sanjuan, M. A., Sequestration of $\mathrm{CO}_{2}$ by concrete carbonation, Env. Sci. Technol. 44 (2010), 3181-3186.

13. Guarguaglini, F. R. And Natalini, R., Fast reaction limit and large time behavior of solutions to a nonlinear model of sulfatation phenomena, Commun. Partial Differ. Equations 32 (2007), 163-189. Zbl1116.35006 MR2304146

14. Hilhorst, D., Issard-Rosch, F. And Roquejoffre, J. M., Large time behavior of the solution to a one-dimensional Stefan problem with a kinetic condition at the free boundary, Euro. Jnl. of Applied Mathematics 15 (2004), 297-313. MR2092915

15. ISHIDA, T. AND LI, C.-H., Modeling of carbonation based on thermo-hygro physics with strong coupling of mass transport and equilibrium in micropore structure of concrete, J. Adv. Concr. Technol. 6 (2008), $303-316$

16. Ishida, T., Maekawa, K. And Solitan, M., Theoretically identified strong coupling of carbobation rate and thermodynamic moisture states in micropores of concrete, J. Adv. Concr. Technol. 2 (2004), 213222.

17. Muntean, A., On the interplay between fast reaction and slow diffusion in the concrete carbonation process: a matched-asymptotics approach, Meccanica 44 (2009), 35-46. Zbl1163.76445 MR2481454

18. Muntean, A. AND BöHM, M., A moving-boundary problem for concrete carbonation: global existence and uniqueness of solutions, J. Math. Anal. Appl. 350 (2009), 234-251. Zb11152.92035 MR2476905

19. Muntean, A. , BÖHM, M. AND Kropp, J., Moving carbonation fronts in concrete: a moving-sharpinterface approach, Chemical Engineering Science 66 (2011), 538-547.

20. Muntean, A., Meier, S., Peter, M., Böhm, M. And Kropp, J., A note on limitations of the use of accelerated concrete-carbonation tests for service-life predictions. Berichte aus der Technomathematik 05-04, Universität Bremen, 2005.

21. Rubinstein, L. I., The Stefan Problem, vol. 27 of Translations of Mathematical Monographs, AMS, Providence, 1971. MR0351348

22. Sanjuan, M. A., Andrade, C., And Cheyrezy, M., Concrete carbonation tests in natural and accelerated conditions, Advances in Cement Research 15 (2003), 171-180.

23. Sisomphon, K., Influence of pozzolanic material additions on the development of the alkalinity and the carbonation behavior of composite cement pastes and concretes, PhD thesis, TU Hamburg-Harburg, 2004.

24. Sisomphon, K. AND FrAnKe, L., Carbonation rates of concretes containing high volume of pozzolanic materials, Cement and Concrete Research 37 (2007), 1647-1653.

25. Souplet, P., Ghidousche, H. And Tarzia, D., Decay of global solutions, stability and blow-up for a reaction-diffusion problem with free boundary, Proc. AMS 129 (2001), 781-792. Zb10959. 35087 MR1802001

26. ThIERY, M., Modélisation de la carbonatation atmosphérique des matériaux cimentaires. Prise en compte des effets cinétiques et des modifications microstructurales et hydriques, Ouvrages d'art OA 52, LCPC, Paris, 2006. 